\title{
Collaborations at the cell front
}

DOI:

10.1038/nrm2359

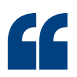

...prompt a

re-evaluation

of current

ideas about

actin filament

organization in lamellipodia.

Electron microscopy of a protruding (left panel) and a slowing (right panel) region at the front of the

lamellipodium. Scale bar represents $100 \mathrm{~nm}$. Image courtesy of S. Koestler and V. Small, Austrian Academy of Sciences, Vienna, Austria.
Current ideas about how protruding lamellipodia are organized have come mainly from electron microscopy (EM) images of cells that show constant motility. Based on EM images that were obtained using a critical-point drying procedure for specimen preparation, a model of lamellipodium organization has been proposed that features a dendritic network of actin filaments with the actin-related protein $2 / 3$ complex situated at 70 -degree branch points. Small and colleagues now reveal a shift in the angular distribution of filaments in lamellipodia according to protrusive activity. These findings shed new light on the way in which actin drives cell motility, and also prompt a re-evaluation of current ideas about actin filament organization in lamellipodia.

Migrating cells exploit two properties of actin filaments to move: the ability to polymerize and push to promote protrusion, and the
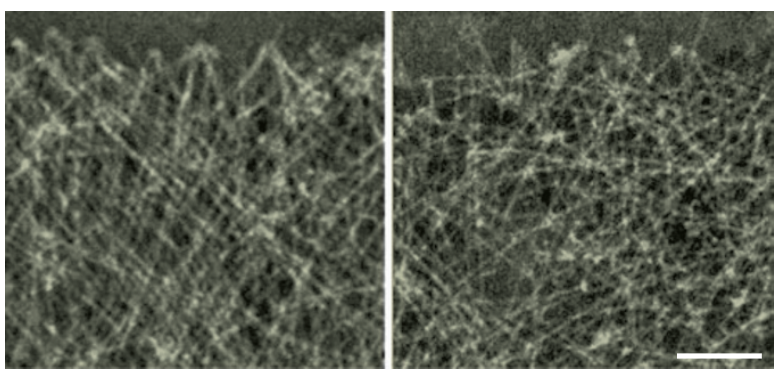

skill to slide with myosin II to drive retraction. Protrusion is achieved by lamellipodia - thin sheets of cytoplasm that are composed of diagonal networks of actin filaments. In migrating cells, lamellipodia undergo phases of protrusion, pause and withdrawal, the latter often being associated with the formation of actin bundles parallel to the cell edge.

To gain insight into the structural basis of changes in protrusive activity, Small and colleagues correlated the local movements of lamellipodia, monitored by live-cell imaging, with their organization after negative-stain EM. They found that actin filaments in protruding lamellipodia subtend at 15-90 degree angles to the front. Transitions from protrusion to pause are associated with a proportional increase in filaments that are orientated more parallel to the cell edge; the authors observed a shift in the orientation of filaments to lower angles (some to $<15$ degrees) and the appearance of a significant proportion of filaments with curved trajectories in pausing zones. The slowing and pause phase is associated with a reduction in filament numbers at the front.

Microspike bundles — bundles that are embedded in the lamellipodia mesh - of actin filaments also showed a wide angular distribution. The proportion of microspikes at lower angles was higher in slowing and pausing lamellipodia by comparison with continuously protruding ones. This angular distribution resulted in a varied velocity of the lateral movement of microspike tips along the cell edge and a variation in the length of the bundles. Microspike bundles moved laterally in opposite directions and could be observed to cross each other or to fuse. These movements promoted the generation of antiparallel arrays of actin filaments that formed bundles at the base of the lamellipodium, from where they entered the lamella and accumulated myosin.

Small and colleagues propose that the angular shift of filaments in lamellipodia helps to adapt to slower protrusion rates, while also maintaining the filament densities that are required for structural support. Single filaments and microspike bundles contribute to the construction of the advancing lamella and to the formation of the cell edge when protrusion ceases. This approach reveals filament arrangements and rearrangements in lamellipodia that are difficult to reconcile with the dendritic model. Which model will survive the test of time remains to be seen.

Ekat Kritikou

ORIGINAL RESEARCH PAPER Koestler, S. A. et al. Differentially oriented populations of actin filaments generated in lamellipodia collaborate in pushing and pausing at the cell front. Nature Cell Biol. 17 Feb 2008 (doi:10.1038/ncb1692) 